\title{
Relação entre permeabilidade e a velocidade de secagem em concretos refratários de alta alumina
}

\section{(Relationship between permeability and drying rate of high-alumina refractory castables)}

\author{
M. D. M. Innocentini', R. G. Pileggi', F. T. Ramal Jr. ${ }^{1}$, \\ A. R. F. Pardo ${ }^{1}$, V. C. Pandolfelli ${ }^{1}$, L. R. Bittencourt ${ }^{2}$ \\ ${ }^{1}$ Departamento de Engenharia de Materiais - DEMa \\ Universidade Federal de S. Carlos - UFSCar \\ Rod. Washington Luiz, km 235, C.P. 676, 13565-905, S. Carlos, SP \\ ${ }^{2}$ Magnesita S.A. \\ Cidade Industrial, 32210-050, Contagem, MG \\ pmmi@iris.ufscar.br,vicpando@power.ufscar.br
}

\begin{abstract}
Resumo
O objetivo deste trabalho foi analisar a relação entre a permeabilidade e a secagem de concretos refratários de alta alumina contendo ultrabaixo teor de cimento, preparados segundo diferentes ajustes granulométricos. Alterações nas distribuições acumulada e na discreta de partículas foram efetuadas variando-se respectivamente o coeficiente de distribuição de Andreasen (q) e o diâmetro máximo de partícula da distribuição $\left(\mathrm{D}_{\max }\right)$, resultando em concretos com diferentes características porosas e fluidodinâmicas. As diferenças foram associadas a parâmetros de empacotamento de partículas no concreto. Os resultados indicaram que o aumento da distância de separação entre as partículas da matriz (IPS) e a diminuição da distância entre as partículas de agregado (MPT) foram responsáveis pelo aumento exponencial observado para as constantes de permeabilidade $\mathrm{k}_{1}$ e $\mathrm{k}_{2}$ e pela redução linear no tempo de secagem dos concretos. Formulações com distribuição acumulada tendo maior coeficiente de Andreasen $(\mathrm{q}=0,31)$ e distribuição discreta com tamanho máximo de grão de $\mathrm{D}_{\max }=4,75 \mathrm{~mm}$ foram as mais permeáveis e tiveram o menor tempo de secagem.
\end{abstract}

Palavras-chave: secagem, permeabilidade, concreto refratário, distribuição granulométrica, empacotamento de partículas.

\begin{abstract}
In this work, the relationship between permeability and drying parameters was evaluated for high-alumina ultra-low cement refractory castables prepared according to different particle size distributions. Variations in the cumulative and discrete distributions were carried out by changing respectively the Andreasen's coefficient $(q)$ and the maximum grain size $\left(D_{\text {max }}\right)$. The performance of 9 different compositions were related to particle packing parameters, such as the separation distances between the fine matrix particles (IPS) and between the coarse aggregate grains (MPT). Results revealed that the simultaneous increase in IPS and reduction in MPT were the causes of an exponential increase in the permeability constants $k_{1}$ and $k_{2}$ of Forchheimer's equation and also of a linear decrease in the drying time. The formulation with higher Andreasen's coefficient $(q=0,31)$ and the maximum grain size $\left(D_{\max }\right)$ of 4,75 $\mathrm{mm}$ were the most permeable and with the lowest drying time.
\end{abstract}

Keywords: drying, permeability, refractory castables, particle packing, size distribution.

\section{INTRODUÇÃO}

A crescente preocupação com a redução de gastos energéticos tem levado o setor de refratários a um grande empenho na melhoria do desempenho térmico e mecânico de seus produtos, seja durante o processamento ou uso em alta temperatura. O recurso mais empregado com essa finalidade tem sido a gradual redução do teor de água e dos ligantes hidráulicos à base de cálcio, promovendo uma maior resistência mecânica e refratariedade. No entanto, essa alternativa tem tornado esses produtos cada vez menos porosos e permeáveis e assim ainda mais sensíveis às condições de tratamento térmico. Particularmente no caso de monolíticos e pré-moldados de grande volume, um aquecimento não adequado tende a levar à pressurização do vapor d'água retido na estrutura pouco porosa, causando eventuais falhas mecânicas ou mesmo a explosão do produto [1-2].

Motivados pelo problema de explosão de estruturas de casas 
e prédios durante incêndios, estudos na área de construção civil foram os primeiros a relacionar a estrutura permeável de um concreto com sua distribuição granulométrica [3-4]. Considerando o concreto como um sistema multifásico (matriz, agregados, cimento, água, poros, etc), diversos parâmetros estruturais têm sido propostos, explicando com relativo sucesso a morfologia e a quantidade de poros em um concreto e as conseqüências para suas propriedades, como permeabilidade, resistência mecânica e tendência à explosão.

Recentemente, o ajuste granulométrico das matérias-primas constituintes do concreto vem sendo investigado de modo sistemático na área de refratários [5], como uma ferramenta promissora para a minimização dos riscos de explosão em formulações de reduzido teor de cimento [6]. No entanto, ainda são escassos na literatura os trabalhos que associam diretamente os parâmetros de distribuição granulométrica e de separação entre partículas com dados de permeabilidade e de secagem de concretos refratários.

Neste trabalho, concretos refratários de alta alumina e ultrabaixo teor de cimento, preparados segundo diferentes ajustes granulométricos, tiveram sua permeabilidade e desempenho durante a secagem comparados. As diferenças fluidodinâmicas que existem entre os fenômenos de percolação do fluido durante um ensaio de permeabilidade e de transporte do vapor de água durante o processo de secagem foram analisadas com base em parâmetros de empacotamento das partículas de matriz e agregados do concreto.

\section{PERMEABILIDADE E ESTRUTURA DO CONCRETO}

Idealmente, um concreto deveria ser considerado um sistema bifásico constituído de um esqueleto de grãos grossos (agregados) preenchido com uma matriz de partículas finas. Geralmente, agregados de alumina eletrofundida apresentam uma certa rugosidade e porosidade aberta, mas são essencialmente impermeáveis. Assim, do ponto de vista fluidodinâmico, o principal trajeto para a percolação de fluidos deveria estar nos espaços existentes entre as partículas da matriz, IPS (Inter-particle Spacing Distance), originados pela presença de água ou pelo empacotamento imperfeito. Contudo, devido às restrições de empacotamento impostas pela superfície do agregado (efeito parede), uma região de maior porosidade e menor tortuosidade, denominada ITZ (Interfacial Transition Zone) é formada na interface matriz-agregado [3-4].

As características dessa região interfacial (ITZ) dependem do tamanho relativo das partículas da matriz e agregados e da ligação química provida pelos hidratos do cimento. Estudos recentes têm sugerido que, devido à menor tortuosidade, os poros na ITZ atuam como atalhos à percolação de ar ou vapor d'água [3-5]. Supõe-se que a proximidade entre as interfaces matriz-agregado favoreça a formação de uma rede de poros conectáveis no concreto, reduzindo a resistência global à permeação de fluidos. Assim, o conceito de distância entre os grãos de agregados (Maximum Paste Thickness, MPT), tornase também importante para a análise da permeabilidade. De uma maneira geral, alterações na formulação do concreto que causem aumento no IPS e na ITZ, ou diminuição no MPT tendem a tornar o concreto mais permeável. A Fig. 1 ilustra o conceito físico de cada um desses parâmetros.

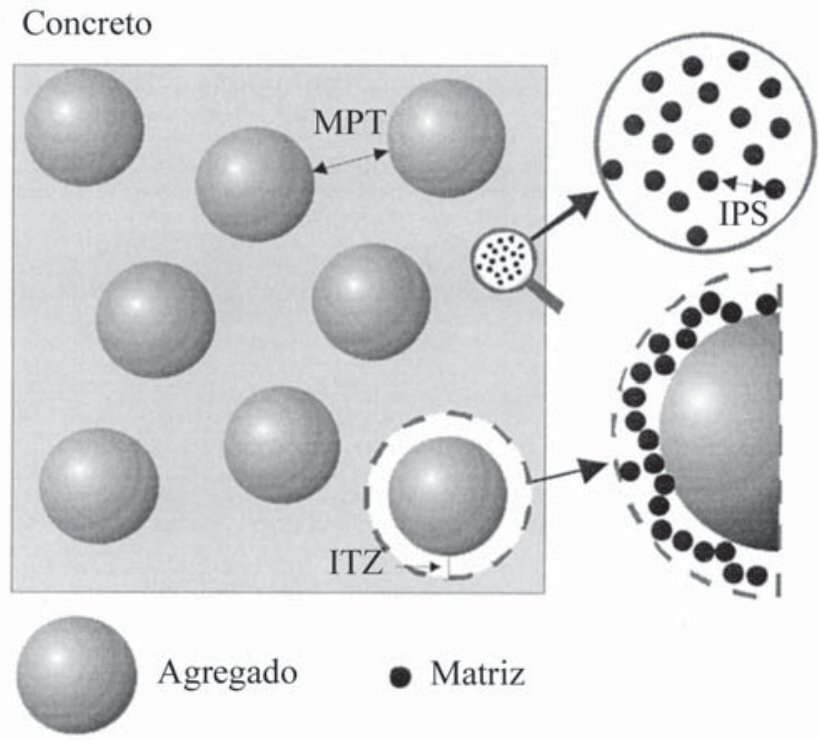

Figura 1: Esquematização da estrutura de um concreto com os parâmetros de separação entre partículas.

[Figure 1: Schematic drawing of the castable structure with particle separation parameters.]

Uma vez que as partículas de matriz e agregados apresentam-se em distribuições de tamanhos, o modelamento matemático realístico desses parâmetros estruturais torna-se complexo, principalmente devido à dificuldade de definição de um diâmetro limite entre grossos (agregados) e finos (matriz) que seja representativo das características fluidodinâmicas do concreto [5]. A investigação experimental de permeabilidade e secagem é assim necessária para a validação e refinamento dos valores calculados de IPS, MPT e ITZ.

Particularmente neste trabalho, foram avaliadas as influências dos parâmetros IPS e MPT, definidos respectivamente como [5]:

$$
\text { IPS }=\frac{2}{\text { VSA }} \times\left[\frac{1}{V_{s}}-\left(\frac{1}{1-P_{\text {of }}}\right)\right]
$$

onde: IPS é a distância de separação interparticular na matriz $(\mu \mathrm{m})$, VSA é a área superficial volumétrica das partículas da matriz, calculada a partir da área superficial específica $\left(\mathrm{m}^{2} / \mathrm{cm}^{3}\right), \mathrm{V}_{\mathrm{s}}$ é a fração volumétrica de sólidos na mistura e $\mathrm{P}_{\text {of }}$ é a porosidade teórica do sistema, quando todas as partículas da matriz estão em contato na condição de máximo empacotamento.

$$
\mathrm{MPT}=\frac{2}{\mathrm{VSA}_{\mathrm{g}}} \times\left[\frac{1}{\mathrm{~V}_{\mathrm{sg}}}-\left(\frac{1}{1-\mathrm{P}_{\mathrm{ofg}}}\right)\right]
$$

onde: MPT é a distância de separação interparticular dos agregados $(\mu \mathrm{m}), V_{\mathrm{gSA}}$ é a área superficial volumétrica dos grossos, $\mathrm{V}_{\mathrm{sg}}$ é a concentração volumétrica dos grossos no concreto e $\mathrm{P}_{\text {ofg }}$ é a porosidade teórica da distribuição de partículas grossas. 


\section{PROCEDIMENTO EXPERIMENTAL}

Concretos refratários de alta alumina e ultrabaixo teor de cimento foram formulados com distribuição de tamanho de partículas ajustada de acordo com o modelo de empacotamento de Andreasen [5], expresso por:

$$
\mathrm{CPFT}=100 \times\left(\frac{\mathrm{D}_{\mathrm{p}}}{\mathrm{D}_{\max }}\right)^{\mathrm{q}}
$$

onde CPFT é a percentagem acumulada de partículas menores que o diâmetro $\mathrm{D}_{\mathrm{p}}, \mathrm{D}_{\max }$ é o diâmetro da maior partícula presente e q é o coeficiente de distribuição da curva granulométrica.

Formulações com 9 diferentes ajustes granulométricos foram desenvolvidas utilizando-se o software PSDesigner [7]. Os coeficientes de Andreasen escolhidos foram $q=0,21$ (para concretos auto-escoantes), 0,26 (para bombeáveis) e 0,31 (para socados), sendo que em cada distribuição acumulada variouse o tamanho máximo de partícula $\left(\mathrm{D}_{\max }=2,80 ; 4,75\right.$ e 8,00 $\mathrm{mm}$ ), mantendo-se constante o tamanho mínimo em $0,1 \mu \mathrm{m}$. Para efeito de cálculo dos parâmetros IPS e MPT adotou-se como diâmetro limite de separação entre matriz e agregados o valor de $100 \mu \mathrm{m}$ [5].

Em todas as formulações, os teores de água (15\% volume) e de cimento ( $1 \%$ peso) foram mantidos constantes. Todas as matérias-primas (aluminas eletrofundidas AEB 4/10 8/20, 40/ F, 10/36 20/40 e 200F, aluminas calcinadas A1000SG e A3000FL e cimento de aluminato de cálcio CA14) foram fornecidas pela Alcoa Brasil e E.U.A. As características gerais de cada composição são apresentadas na Tabela I.

Os corpos-de-prova foram moldados como cilindros de 7,5 cm de diâmetro por $2,5 \mathrm{~cm}$ de espessura para ensaios de permeabilidade e de $4 \mathrm{~cm}$ de diâmetro por $4 \mathrm{~cm}$ de espessura para os ensaios de secagem. A cura foi realizada por 24 horas

Tabela I - Características gerais das composições utilizadas neste trabalho*.

[Table I - General features of the castable compositions studied in this work.]

\begin{tabular}{|c|c|c|c|}
\hline & \multirow{2}{*}{\multicolumn{3}{|c|}{$q=0,21$}} \\
\hline & & & \\
\hline & $\mathrm{D}_{\max }=2,80 \mathrm{~mm}$ & $\mathrm{D}_{\max }=4,75 \mathrm{~mm}$ & $\mathrm{D}_{\max }=8,00 \mathrm{~mm}$ \\
\hline Agregados (\% vol) & 48,8 & 59,8 & 57,8 \\
\hline Matriz (\% vol) & 50,0 & 39,0 & 41,0 \\
\hline $\operatorname{VSA}\left(\mathrm{m}^{2} / \mathrm{cm}^{3}\right)$ & 14,0 & 14,1 & 14,3 \\
\hline $\mathrm{p}_{\mathrm{of}}(-) * * *$ & 18,3 & 18,2 & 18,7 \\
\hline \multirow[t]{3}{*}{$\mathrm{p}_{\mathrm{ofg}}(-) * * *$} & 20,4 & 17,8 & 18,5 \\
\hline & \multicolumn{3}{|c|}{$q=0,26$} \\
\hline & $\mathrm{D}_{\text {max }}=2,80 \mathrm{~mm}$ & $\mathrm{D}_{\max }=4,75 \mathrm{~mm}$ & $\mathrm{D}_{\text {max }}=8,00 \mathrm{~mm}$ \\
\hline Agregados (\% vol) & 59,8 & 64,8 & 67,8 \\
\hline Matriz (\% vol) & 39,0 & 34,0 & 31,0 \\
\hline $\operatorname{VSA}\left(\mathrm{m}^{2} / \mathrm{cm}^{3}\right)$ & 13,5 & 12,7 & 14,2 \\
\hline $\mathrm{p}_{\mathrm{of}}(-) * * *$ & 19,0 & 19,4 & 18,1 \\
\hline $\mathrm{p}_{\text {ofg }}(-) * * *$ & 21,3 & 18,6 & 17,5 \\
\hline
\end{tabular}

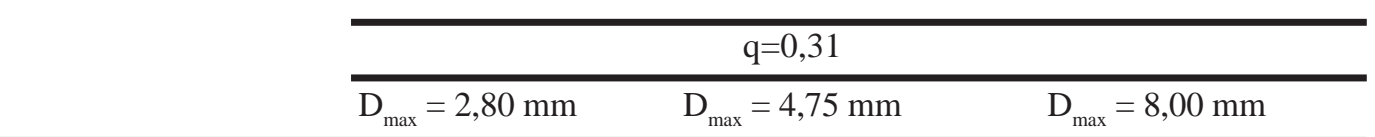

\begin{tabular}{llll}
\hline Agregados $(\% \mathrm{vol})$ & 64,8 & 69,8 & 72,6 \\
\hline Matriz $(\% \mathrm{vol})$ & 34,0 & 29,0 & 26,2 \\
\hline $\mathrm{VSA}\left(\mathrm{m}^{2} / \mathrm{cm}^{3}\right)$ & 12,0 & 12,5 & 12,0 \\
\hline $\mathrm{p}_{\mathrm{of}}(-) * * *$ & 20,5 & 20,7 & 21,1 \\
\hline $\mathrm{p}_{\text {ofg }}(-) * * *$ & 20,5 & 17,7 & 16,1 \\
\hline
\end{tabular}

* Teor de cimento igual a 1,25\%vol (1\% peso) em todas as formulações.

** Assumiu-se VSA $=0$ para os agregados de alumina eletrofundida.

*** Estimado segundo o método de Westman e Hugill [5]. 
em temperatura ambiente $\left(25^{\circ} \mathrm{C}\right)$ e umidade relativa elevada (U.R. 100\%).

Os ensaios de permeabilidade ao ar em temperatura ambiente foram realizados em equipamento descrito anteriormente [8]. Para esta medida, as amostras foram previamente secas a $110{ }^{\circ} \mathrm{C}$ por $24 \mathrm{~h}$ para a eliminação de toda a umidade residual e conversão de hidratos metaestáveis $\left(\mathrm{CAH}_{10}, \mathrm{C}_{2} \mathrm{AH}_{8}\right.$ e $\mathrm{AH}_{3}$ gel) [2]. Os dados experimentais foram ajustados segundo a equação de Forchheimer para o escoamento de fluidos compressíveis [8]:

$$
\frac{\mathrm{P}_{\mathrm{e}}^{2}-\mathrm{P}_{\mathrm{s}}^{2}}{2 \mathrm{P}_{\mathrm{s}} \mathrm{L}}=\frac{\mu \mathrm{v}_{\mathrm{s}}}{\mathrm{k}_{1}}+\frac{\rho \mathrm{v}_{\mathrm{s}}^{2}}{\mathrm{k}_{2}}
$$

na qual $\mathrm{P}_{\mathrm{e}}$ e $\mathrm{P}_{\mathrm{s}}$ são respectivamente as pressões absolutas do ar na entrada e na saída da amostra, L é a espessura da amostra, $\rho$ e $\mu$ são respectivamente a densidade e a viscosidade do ar, $\mathrm{v}_{\mathrm{s}} \mathrm{e}$ a velocidade média do ar, calculada pela divisão da vazão volumétrica pela área frontal da amostra exposta ao escoamento.

Os parâmetros $\mathrm{k}_{1}$ e $\mathrm{k}_{2}$ são conhecidos respectivamente como constantes de permeabilidades Darciana e não-Darciana e são propriedades apenas da estrutura porosa.

Neste trabalho, $\mathrm{k}_{1}$ e $\mathrm{k}_{2}$ foram obtidos por ajuste dos dados experimentais de $\mathrm{v}_{\mathrm{s}}$ versus $\left[\mathrm{P}_{\mathrm{e}}{ }^{2}-\mathrm{P}_{\mathrm{s}}{ }^{2}\right] / 2 \mathrm{P}_{\mathrm{s}} \mathrm{L}$ na equação (D) através do método dos mínimos quadrados. Em cada ensaio, os dados foram coletados em tréplica para garantir a confiabilidade das curvas. Considerou-se $\mu_{\mathrm{ar}}=1,81 \times 10^{-5} \mathrm{~Pa} . \mathrm{s}$ e $\rho_{\mathrm{ar}}=1,08 \mathrm{~kg} / \mathrm{m}^{3}$ para $\mathrm{T}_{\mathrm{amb}}=25^{\circ} \mathrm{C}$ e $\mathrm{P}_{\mathrm{s}}=\mathrm{P}_{\mathrm{atm}}=690 \mathrm{mmHg}$.

Os ensaios de secagem foram realizados em um equipamento termogravimétrico desenvolvido em laboratório, monitorando-se continuamente a temperatura da mufla e a perda de massa da amostra em função do tempo. Os dados foram coletados por dispositivo eletrônico em intervalos de 5 segundos. Para permitir a comparação adequada dos resultados, os corpos também foram previamente aquecidos por 24 horas a $110^{\circ} \mathrm{C}$, resfriados e posteriormente saturados em água por 2 horas sob vácuo para o preenchimento de toda a porosidade aberta. A taxa de aquecimento de $12{ }^{\circ} \mathrm{C} / \mathrm{min}$ foi constante em todos os ensaios no intervalo de 25 a $800{ }^{\circ} \mathrm{C}$ com patamar de 60 min a $800^{\circ} \mathrm{C}$. Os parâmetros de análise foram a temperatura da mufla (Temperatura ${ }_{50 \%}$ ) e o tempo de ensaio (tempo ${ }_{50 \%}$ ) necessários para que os corpos perdessem metade de toda a massa de água presente, incluindo hidratos e água livre.

\section{RESULTADOS E DISCUSSÃO}

A Fig. 2 mostra que, para composições com um mesmo $\mathrm{D}_{\max }$, a permeabilidade dos concretos variou exponencialmente com o coeficiente de distribuição de Andreasen. Uma alteração em q de 0,21 para 0,31 ocasionou um aumento médio de 3 vezes em $\mathrm{k}_{1}$ (Fig. 2a) e de 20 vezes em k $\mathrm{k}_{2}$ (Fig. 2b). No entanto, observa-se que dentro de uma mesma distribuição acumulada, a variação em $D_{\max }$ de 2,80 para $8,00 \mathrm{~mm}$ também causou o aumento, embora de intensidades distintas, nas constantes $\mathrm{k}_{1} \mathrm{e}$ $\mathrm{k}_{2}$. A composição com $\mathrm{D}_{\max }=4,75 \mathrm{~mm}$ foi a mais permeável, indicando não ser direta a relação entre o aumento do tamanho máximo de grão e o aumento de permeabilidade do concreto.
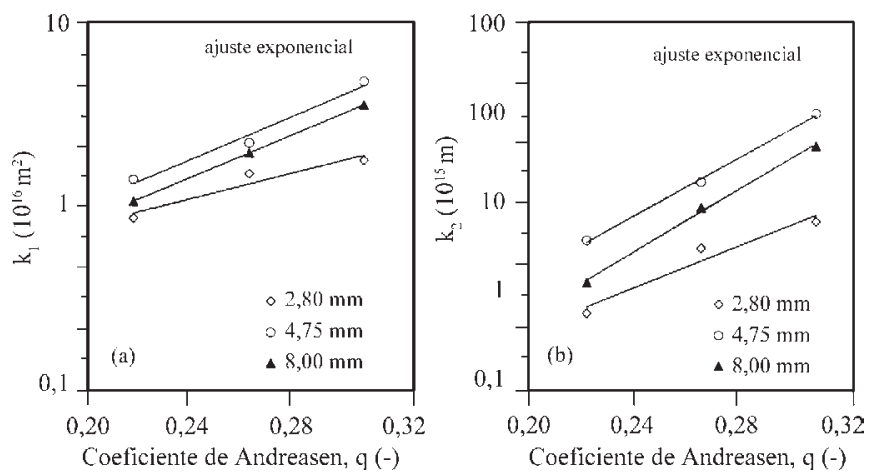

Figura 2: Influência da distribuição acumulada (q) e da distribuição discreta $\left(\mathrm{D}_{\max }\right)$ sobre as constantes de permeabilidade dos concretos estudados.

[Figure 2. Influence of cumulative $(q)$ and discrete $\left(D_{\max }\right)$ particle size distribution parameters on the permeability constants of the tested castables.]

Este resultado reflete a grande dificuldade prática na escolha da distribuição granulométrica de uma composição cerâmica como base para ajuste nas propriedades físicas do concreto. Ajustes granulométricos baseados em uma mesma distribuição acumulada de partículas (curvas log-log de CPFT versus $\mathrm{D}_{\mathrm{p}}$ com mesma inclinação q) podem gerar corpos com propriedades totalmente distintas dependendo da distribuição discreta. Em geral, a literatura tem verificado que existe uma associação clara entre as propriedades de composições com distribuições acumuladas diferentes e mesma distribuição discreta, embora a recíproca não seja necessariamente verdadeira [5-6, 8], o que se torna um problema ainda mais sério na indústria considerando a variabilidade de tamanhos encontrada entre lotes de uma mesma matéria-prima.

Na verdade, os resultados na Fig. 2 confirmam a necessidade
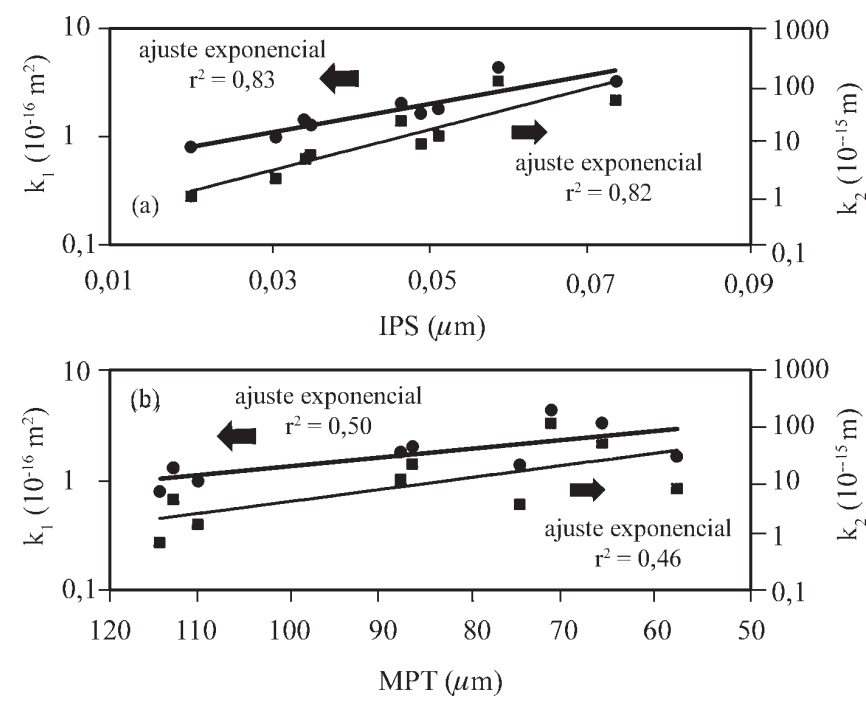

Figura 3: Influência dos parâmetros IPS e MPT sobre as constantes de permeabilidade dos concretos estudados.

[Figure 3: Influence of parameters IPS and MPT on the permeability constants of the tested castables.] 
de parâmetros que especifiquem as alterações físicas na estrutura do concreto causadas pelas variações nas distribuições discreta e na acumulada, permitindo uma correlação mais genérica e segura com dados de permeabilidade e secagem. Nesse sentido, a Fig. 3 mostra a relação entre os parâmetros IPS e MPT com as constantes $\mathrm{k}_{1}$ e $\mathrm{k}_{2}$.

A Fig. 3 mostra que houve uma relação exponencial forte entre a distância de separação das partículas da matriz (IPS) e as constantes de permeabilidade $\mathrm{k}_{1}$ e $\mathrm{k}_{2}$. Esse resultado indica que as alterações efetuadas nas distribuições acumulada e discreta causaram na verdade uma maior abertura dos poros da matriz, facilitando a percolação do ar. Tal fenômeno pode ser entendido considerando-se que o aumento no coeficiente de Andreasen q corresponde na prática a um aumento na proporção agregado/ matriz, com a diminuição na distância entre os agregados (MPT) e consequentemente uma maior aproximação entre as regiões interfaciais no concreto (ITZs), consideradas pela literatura como menos resistentes à percolação de fluidos. Além disso, uma vez que os agregados podem ser considerados pouco ou mesmo impermeáveis, tem-se que uma maior quantidade de água (mantida em teor constante nas formulações) é disponibilizada para a separação das partículas finas na matriz. Neste caso, o cimento tem papel importante na manutenção dos canais permeáveis na matriz, através da rede de hidratos que evita a aproximação entre as partículas após a remoção da água, explicando os aumentos de permeabilidade nas Figs 2 e 3 a.

Em relação ao efeito do $D_{\max }$, tem-se que o aumento no tamanho máximo de grão proporciona um aumento na quantidade (em volume) de agregados no concreto, porém com a diminuição do número de partículas. Como consequiência, embora a composição com $\mathrm{D}_{\max }=2,8 \mathrm{~mm}$ apresente os menores agregados (poros interfaciais mais curtos na ITZ), ela apresenta um maior número de partículas grossas. No outro extremo, a composição com $\mathrm{D}_{\max }=8,00 \mathrm{~mm}$ tem o maior volume de agregados, porém proporcionalmente com o menor número de interfaces permeáveis. Esse efeito oposto sobre a permeabilidade explicaria o fato da composição com tamanho máximo de grão intermediário $\left(D_{\max }=4,75 \mathrm{~mm}\right)$ apresentar na Fig. 2 os maiores níveis de permeabilidade para todas as distribuições acumuladas estudadas. De qualquer modo, a Fig. $3 \mathrm{~b}$ mostra que, independentemente do tipo de alteração na distribuição granulométrica do concreto ( $q$ ou $\mathrm{D}_{\max }$ ), a diminuição no MPT causou aumento nas constantes de permeabilidade $\mathrm{k}_{1}$ e $\mathrm{k}_{2}$. Contudo, a correlação não tão forte $\left(\mathrm{r}^{2}=0,50\right.$ e 0,46 respectivamente) confirma que não somente a variação no MPT deve ser considerada, mas também o tamanho (comprimento) dos poros interfaciais na ITZ.

Tendências mais nítidas foram observadas na Fig. 4 em relação aos ensaios de secagem. Houve uma forte correlação linear $\left(r^{2}=0,91\right)$ entre o parâmetro IPS e a temperatura atingida após a remoção de $50 \%$ da água no concreto (Fig. 4a), confirmando que o aumento de permeabilidade ao ar foi também efetivo para o transporte mais rápido de vapor d'água, e consequentemente para a diminuição do tempo de secagem. $\mathrm{O}$ aumento na distância interparticular de 0,02 para $0,07 \mu \mathrm{m}$ proporcionou uma diminuição de $50{ }^{\circ} \mathrm{C}$ (de $302^{\circ}$ para $253^{\circ} \mathrm{C}$ ) em $\mathrm{T}_{50 \%}$, indicando um menor risco de pressurização do vapor d'água e um ganho efetivo no tempo de secagem do concreto.
A Fig. 4b mostra que o parâmetro MPT também apresentou um ajuste linear claro com a temperatura de secagem dos concretos. Ainda assim, não foi obtido um coeficiente de correlação tão alto $\left(r^{2}=0,68\right)$ como o observado para o IPS $\left(r^{2}\right.$ $=0,91)$ na Fig. $4 \mathrm{a}$.

Um fator importante a ser considerado na análise dos dados nas Figs. 3 e 4 é que as variáveis escolhidas neste trabalho para as alterações granulométricas dos concretos $\left(\mathrm{q}_{\text {e }} \mathrm{D}_{\max }\right)$ foram responsáveis por variações simultâneas nos parâmetros IPS, MPT e possivelmente em ITZ. Assim, embora tenham sido claras as relações entre esses parâmetros e os dados de permeabilidade e de secagem, não foi possível distinguir a contribuição individual das características da matriz (IPS) e dos agregados (MPT e ITZ) para o aumento das constantes $\mathrm{k}_{1}$ e $\mathrm{k}_{2}$ e para a diminuição em $\mathrm{T}_{50 \%}$.
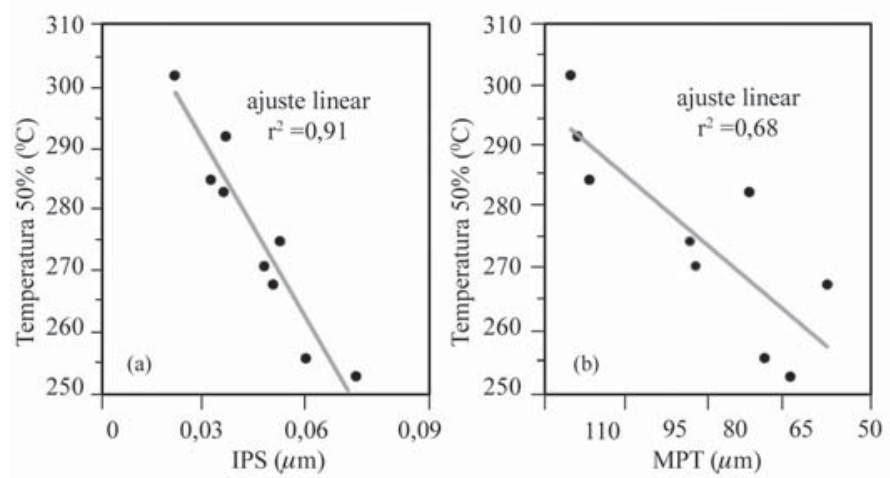

Figura 4: Influência dos parâmetros IPS e MPT sobre a temperatura necessária para a remoção de $50 \%$ da água dos concretos estudados. [Figure 4: Influence of parameters IPS and MPT on the temperature required to remove $50 \%$ of water from the tested castables.]

No entanto, os resultados deste trabalho revelaram claramente que a estrutura porosa afeta de modo diferenciado a permeação de ar e de vapor d'água através do concreto. Enquanto as constantes de permeabilidade $\mathrm{k}_{1}$ e $\mathrm{k}_{2}$ tiveram uma variação exponencial com o IPS e MPT, a temperatura de secagem, e com isso o tempo de secagem, tiveram uma dependência apenas linear. A conseqüência dessa tendência pode ser observada na Fig. 5, que mostra uma relação inversa
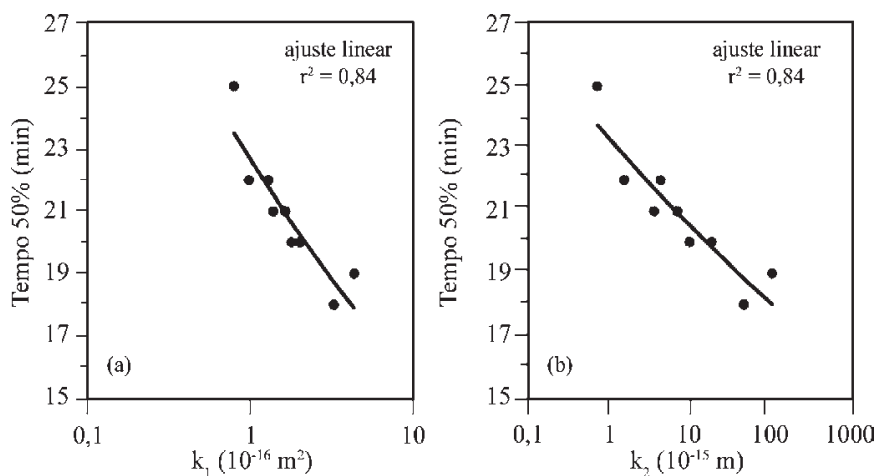

Figura 5: Relação entre constantes de permeabilidade e tempo de secagem para os concretos estudados.

[Figure 5: Relationship between permeability constants and drying time for the tested castables.] 
entre constantes de permeabilidade e o tempo para a remoção de $50 \%$ da água do corpo. Na prática, nas condições de ensaio deste trabalho, as alterações realizadas na distribuição granulométrica causaram uma variação relativa de até 7 minutos na secagem das amostras.

As causas desse comportamento ainda devem ser melhor analisadas, mas podem estar relacionadas às diferenças fluidodinâmicas entre o trajeto do ar e do vapor d'água nos ensaios de permeabilidade e de secagem, respectivamente. Assim, em relação à permeabilidade, a diminuição do MPT reduz a distância entre as interfaces permeáveis do concreto, enquanto o aumento simultâneo do IPS facilita a percolação do ar pela matriz que separa as interfaces, potencializando o aumento de permeabilidade. Por outro lado, espera-se que, durante a secagem, o vapor d'água gerado tenha seu transporte principalmente dominado pelas características da matriz, em cujos poros está retida a maior parte da água no concreto. Também deve ser considerado que o fenômeno de secagem é mais complexo, e depende, além da permeabilidade, de vários outros mecanismos de transporte, como condução térmica, difusão mássica, capilaridade e convecção.

De qualquer modo, os parâmetros de empacotamento IPS e MPT mostraram neste trabalho que composições com matrizes mais porosas e com maior aproximação entre os agregados são mais permeáveis e apresentam um tempo menor de secagem. Nesse sentido, as formulações com distribuição acumulada tendo maior coeficiente de distribuição de Andreasen tiveram melhor desempenho. Por outro lado, para uma mesma distribuição acumulada, o aumento no tamanho máximo de grão favoreceu até certo ponto o processo de secagem, sendo a amostra com $\mathrm{q}=0,31$ e $\mathrm{D}_{\max }=4,75 \mathrm{~mm}$ a de melhor desempenho global neste trabalho.

\section{CONCLUSÕES}

O objetivo deste trabalho foi analisar os desempenhos de permeação e de secagem de concretos refratários de alta alumina e ultrabaixo teor de cimento preparados segundo diferentes ajustes granulométricos. Os resultados mostraram uma clara relação dos parâmetros de empacotamento de partículas da matriz e dos agregados com as constantes de permeabilidade e com a temperatura de secagem. O aumento exponencial observado para as constantes $\mathrm{k}_{1}$ e $\mathrm{k}_{2}$ foi explicado com base no aumento simultâneo da distância de separação entre as partículas da matriz (IPS) e diminuição da distância entre as interfaces matriz agregado (MPT). Por outro lado, houve uma relação linear entre os parâmetros IPS e MPT com a temperatura de secagem $\mathrm{T}_{50 \%}$. De um modo geral, as formulações com distribuição acumulada tendo maior coeficiente de Andreasen $(q=0,31)$ e distribuição discreta com tamanho máximo de grão intermediário $\left(D_{\max }=4,75 \mathrm{~mm}\right)$ foram as mais permeáveis e tiveram o menor tempo de secagem.

\section{AGRADECIMENTOS}

Os autores agradecem a FAPESP, Magnesita S.A. e Alcoa Alumínio S.A. pelo apoio na realização deste trabalho e aos alunos L. A. Nascimento e A. C. Rizzi Jr. na condução dos experimentos.

\section{REFERÊNCIAS}

[1] W. E. Lee, R. E. Moore, "Evolution of in-situ refractories in the 20th century", J. Am. Ceram. Soc. 81, 6 (1998) 13851410 .

[2] R. E. Moore, J. D. Smith, W. L. Headrick, T. P. Sander, "Monolithic dewatering theory testing and practice: new challenges", In: 32nd Annual Symposium on Refractories, The Am. Ceram. Soc. St. Louis Section, (1996) 26.

[3] K. L. Scrivener, K. M. Nemati, "The percolation of pore space in the cement paste/aggregate interfacial zone of concrete", Cement Concrete Res. 26, 1 (1996) 35-40.

[4] D. P. Bentz, E. J. Garboczi, P. E. Stutzman, "Experimental and Simulation Studies of the Interfacial Zone in Concrete", Cement Concrete. Res. 22, 5 (1992) 891-902.

[5] I. R. Oliveira, A. R. Studart, R. G. Pileggi, V. C. Pandolfelli, "Dispersão e empacotamento de partículas - Princípios e aplicações em processamento cerâmico", Fazendo Arte Editorial (2000) 224.

[6] M. D. M. Innocentini, A. R. Studart, R. G. Pileggi, V. C. Pandolfelli, "PSD effect on the permeability of refractory castables", Am. Ceram. Soc. Bull. 80, 5 (2001) 31-36.

[7] R. G. Pileggi, F. Ortega, R. Morabito, S. L. Vendrasco, V. C. Pandolfelli, "Desenvolvimento e aplicação de um software que automatiza o processo de combinação de matérias-primas na obtenção de produtos cerâmicos", Cerâmica 44, 289 (1998) 189-195.

[8] M. D. M. Innocentini, A. R. Studart, R. G. Pileggi, V. C. Pandolfelli, "Permeabilidade de concretos refratários multifuncionais", Cerâmica 47, 301 (2001) 34-39.

(Rec. 19/03/02, Ac. 28/06/02) 